\title{
User Centered Design for Battlefield Screen
}

\author{
Heesoo Jung1, Suhwan Kim², Yongjin James Kwon ${ }^{1 *}$ \\ ${ }^{1}$ Department of Industrial Engineering, Ajou University, Suwon, South Korea \\ ${ }^{2} 1^{\text {st }}$ Division, $3^{\text {rd }}$ Department, Agency for Defense Development, Daejeon, South Korea \\ Email: "yk73@ajou.ac.kr
}

Received 11 May 2016; accepted 19 May 2016; published 26 May 2016

\begin{abstract}
This thesis paper is a brief summary of user centered design, (particularly in the situation of under the battlefield), which incorporates the related information after reviewing many other papers. A display screen user requires an ability to provide proper information while considering a variety of information in a short period. While operating the display screen, the user is heavily pressed for time as the missions are allocated through many different steps of orders. In addition, since a failure would cause fatal damage or cause further defeat, this increases the pressure of time. The right environment for operating a display screen is most useful when the user is in the most vulnerable time period or long-term continuous operation of the display screen. In that kind of sterile environment, the operating display screen is there to help the user's decisions for the matter at hand, in this it should follow the user centered design and improve the user's handiness and use. Furthermore, the information should be provided in accordance with the user's informational and perceptional process, and the capacity of the information process should be improved with the movement through each process and step. This thesis paper can be used as a reference material when designing the display screen in order to apply a user's experiences.
\end{abstract}

\section{Keywords}

User Centered Design, Interface Design, Software Engineering, Battlefield Screen, Operating Display

\section{Introduction}

The design of the display screen is meant to improve the user's use. With all design processes, the user and designer aim at designing an environment that applies that user's experiences through a mediation and compromise. That will improve the ease of use of the display screen. For example the design of the display screen would reduce the user's time to learn the environment and decreases operational errors. It also cuts down task incapability when irresistible exigent circumstances arise. This kind of designing method called "User Centered Design". User's comments and data of evaluations contributes making better display screen. There are several requirements for participating in a designing process for a display screen, is below (Table 1).

We expects several effects by using user centerd design methodologies. It is to help user's decision making and decrease user's burden caused by hard circumstances, such that below (Table 2) [1].

\footnotetext{
"Corresponding author.
} 
Table 1. Members of user centered design process.

\begin{tabular}{|c|c|}
\hline Member & To do \\
\hline Designer & $\begin{array}{l}\text { - } \quad \text { Seize product purpose and intention } \\
\text { - Grasp the capacities and details when designing the method of operation } \\
\text { - } \text { Consider the welfare and convenience of the user } \\
\text { - Reflect the user's evaluation consistently }\end{array}$ \\
\hline User & $\begin{array}{l}\text { - Express the purpose for using the product } \\
\text { - Check every step of design and express any opinions } \\
\text { - Reflect the opinions in the usual user environment } \\
\text { - Participate in the design }\end{array}$ \\
\hline
\end{tabular}

Table 2. Effects of user centered design.

\begin{tabular}{cl}
\hline Circumstance & \\
\hline Urgent & - Consider conveying information beyond human information loading \\
situation & - Minimize errors \\
& - Use the suitable auxiliary equipment \\
$\begin{array}{c}\text { Education } \\
\text { situation }\end{array}$ & - Minimize the learning time by designing it with social norms in mind \\
& - Improve the user's convenience \\
Iterative & - Improve the user's mental satisfaction \\
operation & - Improve the efficiency of conveying information \\
& - Minimize operational errors \\
\hline
\end{tabular}

\section{Principles of Operational Interface Design}

Fundamentally, when a user conforms to a software interface, one gets used to it through one's experience with it. Thus, when the designer plans for an operational function and structure of an interface, one needs to consider the user's prior knowledge. The basic interface structure principle, that reflects the user's experience, is like below:

- Having the procedural structure for a performance that is identical to the user's operational process.

- Designing an analogical UI manipulation to mimic a similar operational execution to the user's experience.

- Structures, which are in between hierarchical interfaces, should have the same user's experienced knowledge.

- Existing efficient manipulation of the users.

- Minimizing the diversity and composition of interface manipulation and operation.

There are criteria of "intentional agreement" and "operational efficiency" to examine whether the designer truly applies the user's experience to the design. The intentional agreement is a criterion to check how much the user's opinions agree with the design; the operational efficiency is to calculate how many steps a software interface needs to execute a task. The higher the scales in both intentional agreement and operational efficiency, the more reflective they are of the user's intentions, and the closer to an efficient software interface. To improve the intentional agreement, first, both the user and designer must describe the steps to perform a single execution separately. After that, the designer can conform the motions to the steps that the user stated. In order to enhance the operational efficiency, the motions from each execution in the motion charts that are in common should be combined, whereas unessential motions should be eliminated [2].

First, the components of the information that affects communication performance. According to a study on the properties to visualize information, components of the information can be divided into content, structure, format. Contents can be classified based on importance in very critical information, needed information, minor information in a particular situation. Structure is a property of the information represented or organized method. The format is a way to highlight or represent information. It is possible to improve the information delivery performance by controlling the components in the information purposes. Second, expression of the visualization information affects communication performance. Another method to visualize information, there are ways to take advantage of an icon, symbolism or infographics, and typography [3]. 


\section{Process of User's Experience Centered Design}

When the designer applies the user's experience in the software interface of the national defense display screen, it is quickest to utilize the user centered design process in the beginning of the design. This helps to save a lot of cost in the examination process. The below is the Alan Cooper's user experience design process (Table 3) [4] [5].

- First, understand the product and the user's trait and purpose- what kind of uses the product has, what kind of duty it would perform, what the user's role is, and who would use the product.

- Second, make a scenario for the product use-the more scenarios are conditionally made, the less product errors occur in the actual usage after the completion.

- Third, know necessary information and needs when executing the newly created scenario to fulfill the duty.

- Fourth, design the product's hardware, software, and interface.

- Last, improve or reform it based on the capacity evaluation and the user's evaluation. Repeating the evaluations and reformations will promote the product's perfection.

\section{Design Reflecting the User's Information Cognition Process}

The national defense display screen should consider the user's information cognition process in order to efficiently transfer massive data to the user in a short period of time. A user's information cognition is the process concerned with utilizing and distributing the limited human intellect. A user's attention needs in every process to recognize and manage information; thus, if it is dispersed in too many places, one can have hard time understanding it fully and instead try harder to concentrate on gathering all the information separately, which would make the user tired more quickly. Thus, it is important to distribute the information with regards to the human intellect appropriately. A human being's memory divides into long-term and short-term memories: the shortterm memory remains for a number of seconds and it is calculated at the speed of light, and the long-term memory is proficient, natural thoughts by repeated experiences. To utilize the user's short-term memory efficiently, data should be provided in chunks. It is the most efficient to memorize information that is 5 to 9 (average 7) units long. In contrast, in order to use a human's long-term memory efficiently, it is important to provide the information as related to a human instinct. Then, the user can quickly compute the information that is in one's long-term memory [6].

\section{Design Reflecting a Human's Information-Processing Process}

The human information process is used to handle all the processes that are made up of a human's stimulation, this consists of information perception, recognition, decision-making, and planning for the action and behavior. According to Jens Rasmussen, a human's information-processing consists of eight processes and its organic movements for each [7].

The eight processes consist of 1) activation, 2) observation, 3) understanding the situation, 4) analysis of a result, 5) comparison and evaluation, 6) motion definition, 7) design a process, and 8) performance. The activation phrase is used to get a motivation to complete a user's purpose or goal. It is important to recognize the necessity of selecting received information and making a meaningful decision in the national defense structure.

Table 3. User centered design process (Cooper A, 2010).

\begin{tabular}{ccc}
\hline No & Process & Explanation \\
\hline 1 & Research Understand & the user and project \\
2 & User Model & User and project scenario \\
3 & Deriving the needs & Specific, necessary information \\
5 & Design & Design plan \\
6 & Edition & Manipulating Interface procedure or improving content
\end{tabular}


The observation phase is used to select and execute the right, necessary data and variables among a variety of information given from the user's display screen.

Next, one should define the interior system situation from the selected data and variables in this third phase.

In the analysis phase, it is effective to enhance completeness by repeating a comparison and evaluation with the patterns from a user's long-term memory that have already become a skilled, operational process.

The motion defining phase is to prepare for adjusting actions and behaviors to achieve the goal or purpose which is set in the beginning of the process.

The last phase is for completing the adjustment precisely with the determined process and procedure.

All phases stated above basically require a display screen to efficiently provide the information. Any similar information should be organized and minimized in the display screen in order not to distract the user's attention and memory. In addition, each phrase should help the user make the right decision while providing proper information at a suitable time so that the user's decision is the best option among all others. Below are additional facts that should be considered in providing the information for each step.

- A composition of the information, menu and the information display for the activation, observation, understanding the situation, analysis of the result, evaluation, defining the project, making the procedure, and performance.

- Defining the user's necessity accurately in the activation phrase.

- Selecting a variable and necessary data to fulfill the purpose in the observation phase.

- Providing additional information to the user to understand the interior systematic situation in the understanding phase.

- Providing additional information to the user to help make the right decision in the decision making phase.

- Providing an exact standard to the user's decision making in the evaluation phase.

- Providing visual aids that are necessary to the system in the motion defining phase.

- Providing visual aids to make it easily understandable in the designing phase.

- Helping the user to reduce errors while executing in the performing phase.

\section{Evaluation Index or Rubric to Optimize Convenience and to Derive a Useful Evaluation}

One can measure convenience by the level of satisfaction the user feels when one uses the software product or operates the system. It is also a measurement tool to evaluate whether the product applies a physical and emotional pressure while using the program repeatedly for a long period of time and to check whether that causes any deterioration in the product. The below chart (Table 4) shows the criteria for evaluating the user's convenience while using a digital interface [8]:

In going over the chart, here is a summary of the effects that influence the user's convenience.

- Whether it is easy to recognize the information within the interface.

- Whether it is easy to understand the information within the interface.

- Whether it is easy to use the interface.

Table 4. Usability estimation index for digital interface (Wang H, Xue C and Wang Z, 2010).

\begin{tabular}{ccc}
\hline \multicolumn{1}{c}{ No } & Usability Mectrics & Measuring Index \\
\hline Easy to perceive the interface information & & Efficiency \\
Easy to understand the interface information & Speed \\
Easy to remember the interface information & Keeping time \\
Easy to learn how to use the interface & Assists \\
Less errors & Errors \\
Aestheticexperience in vision and auditory sense & Subjective rating \\
\hline
\end{tabular}


- Whether it is easy to memorize the information in the interface.

- Whether it prevents the user from operating errors.

- Whether it helps cope with the errors easily after they occur easily.

- Whether the visual aid and hearing feedback from manipulating the information are artistically pleasing.

- Whether it causes the user a psychologically positive experience when operating it.

The usefulness is divided into four categories to be an index to evaluate the mutual capacity between a human and an interface. First, if it improves the probability of success to complete a duty by decreasing running time or operating errors in the interface, if it brings the user in line with the preciseness of operation, and if the capacity of having a quick latent period or response time is good enough when the interface and the user face each other. Second, the interface evaluates the user's level of satisfaction. Evaluating factors are measured by a subjective survey of the user. Third, the interface evaluates the user's corresponding level of physical features. The factors are measured by the user's physical index and behavioral index. Last, it is assessed by a cognitive psychological theory which measures the interaction standard between the user and the interface.

- Evaluate the probability of success, probability of error, running time, and the contribution level to the duty about the user and interface's contact.

- Let the user use the charts (self-evaluation chart and the measurement level chart) to measure the degrees to evaluate the usefulness of the interface subjectively.

- Use psychological and behavioral index measurements, pupil chasing data, electromyogram-electrocardiogram (ECG) data, and thermogram data to assess the response of the user.

- Use the cognitive psychological theory of GOMS (Goals, Operators, Methods, Selections rules) that calculates conversational operating completion time in order to assess the measurement of the interaction between a human and interface.

\section{Conclusions}

The design should consider adaptability with expectations that are based on the user's experience, purpose, intention, and concept while designing a user centered computer software. The computer software interface should be analogical to the user's experience and have socially used methods for any interface. The interface should also functionally have a similar executing process to the user's way of thinking. The designer also needs to improve the interface while considering the purpose for its use by doing consistent research. There are basic things for the designer to consider maintaining smooth communication between the user and the interface. The designer should maintain a natural executing ability with a procedure based on the user's knowledge and environmental information that the user utilizes when operating the software interface. Complexity in the interface menu increases confusion and the probability of error so the user is likely to be unproductive with such an interface. Therefore, as long as it does not interrupt the user, the interface should be created in accordance to the user's duty performing process, which should also fit simply to the ideas and concepts that the user has. When the designer creates an interface, they should keep in mind how to help the user learn to operate the interface. If the user takes a lot of time to learn the software interface, that means the interface is not friendly - this will take a lot more time for the user to get used to and will also possibly cause errors. very step of the operating interface should anticipate the user's possible use function to minimize all those potential errors. This will prevent the user from causing fatal errors in the interface and that also agrees with the economical principle of helping to minimize the number of functions to the least possible.

- Add the additional information collected by regular research about the user's purpose so that the information in the software interface can be matched to it.

- Design the interface menu with the user's idea and/or functional processes in mind.

- Consider whether the interface functionally helps the user enough to complete any duty performance the user has.

- Consider that any control is necessary to be created if the user has to control it by onesself in every step of the interface.

- Evaluate the adaptability of the interface for the user by measuring convenience and usefulness.

- Analyze and improve consistently by conducting repeated examinations to fulfill the user's satisfaction and to create a suitable interface for the user.

- Consider the methods used to teach the user how to execute the interface effectively. 
- Consider if any tutorial or auxiliary method is necessary to be used to have the user understand the interface better.

- Understand the limitations of a human's attention and memory resources and distribute information properly.

- Provide the information in conformity to the organic linkage within a human's information processing step.

- Enhance the capacity of processing information in the phase of a human's information processing.

\section{Acknowledgements}

This work was supported by the Agency for Defense Development (ADD) under the Contract No. UD140066CD. The authors wish to express sincere gratitude for the financial support.

\section{References}

[1] Lee, S. (1999) Research for Evaluating Usability Using WWW (World Wide Web). Master's Thesis, KAIST, Daejeon.

[2] Vicente, K.J. (1999) Cognitive Work Analysis-Toward Safe, Productive, and Healthy Computer-Based Work. CRC Press.

[3] Yoon, M. and Yoon, W. (2006) Development of Interaction Design Draft for User Interface Design Assessment Process Support. JESK, 353-358.

[4] Lee, J. and Koo, M. (2013) A Study on the Design Process based on User Experience (UX). KODDCO, 13, $485-494$.

[5] Cooper, A. (2007) About Face 3: The Essentials of Interaction Design. Willy.

[6] Wang, H., Xue, C. and Wang, Z. (2010) The Quantitative Usability Evaluation of Fighter Cockpit Digital Interface CAIDCD. 2010 IEEE 11th International Conference on, 1, 384-387.

[7] The Decision Ladder Template. Adapted from Rasmussen, J. (1976) Outlines of a Hybrid Model of the Process Plant Operator. In: Sheridan, T.B. and Johannsen, G., Eds., Monitoring Behaviour and Supervisory Control, Plenum, New York, 371-383, with Permission, and from Rasmussen, J., Pejtersen, A.M. and Goodstein, L.P. (1994) Cognitive Systems Engineering. Wiley, New York, with Permission.

[8] Wang, H., Xue, C. and Wang, Z. (2010) The Quantitative Usability Evaluation of Fighter Cockpit Digital Interface CAIDCD. 2010 IEEE 11th International Conference on, 1, 384-387. 